\section{Colony PCR Amplification of Actinomycete DNA}

Sir:

Resistance to antibiotics has made the treatment of human infectious diseases increasingly more difficult, with many pathogenic bacteria now completely resistant to all useful antibiotics. Actinomycetes are a group of Grampositive, filamentous bacteria well known as a source of antibiotics. Isolation of actinomycetes representing rare genera from previously unexplored niches or biodiversity hotspots has become an important bioprospecting strategy in our search for new antibiotics. Likewise, interchanging secondary metabolism genes between actinomycetes to create unnatural gene combinations may also yield useful antibiotics in the future ${ }^{1)}$. Genetic techniques to select for such actinomycete genera or biosynthetic genes have been hampered by the lack of an efficient and rapid DNA extraction procedure amenable to high sample throughput. This is because the composition and complexity of actinomycete cell walls vary considerably between different genera compared to other Gram-positive bacteria. Here we describe a rapid and efficient extraction procedure yielding DNA of sufficient quality suitable for high throughput screening of actinomycetes by PCR.

The following actinomycetes were used in this study: Amycolatopsis mediterranea NCIMB 9613, Mycobacterium avium (strain $\mathrm{A}+$, Institut Pasteur, Paris, France), Nonomuraea pusilla NCIMB 11116, Nonomuraea roseoviolacea subsp. roseoviolacea NCIMB 11117, Nonomuraea ferruginea NCIMB 11630, Nonomuraea roseola NCIMB 11632, Saccharopolyspora erythraea NRRL 2338, Streptomyces antibioticus NCIMB 11506 and Streptomyces cinnamonensis NCIMB 12604. All strains were cultured following instructions from the culture collections. Through multiple sequence alignments, differences in nucleotide sequences of $16 \mathrm{~S}$ rRNA genes from a range of actinomycetes were used to design two pairs of universal and genus-specific primers ${ }^{2}$. The universal primers were, in the forward direction 5'-TGCGGGTCTGCGGTGGATACGGGC- $3^{\prime}$ and in the reverse direction 5'-ATCGCCAGCCCCACCTTCGACCGCT- $3^{\prime}$. Primers specific for the genus Nonomuria had the following forward sequence 5'TGGTGGTGGAAAGTTTTTTCG-3' and reverse sequence
5'-GCAGACCCGCAGTTAAGCTG-3'. In addition, two PCR primers were specifically designed to amplify a 1358 bp region from the erythromycin producing polyketide synthase of S. erythraea NRRL 2338 (GenBank accession number M63676, nucleotide position 2256 to 3614): forward primer 5'-GAACCGGTCGCGGTCGTC-3', reverse primer 5'-CAGACCTTCCGCGCTGCTC-3'. Typically, a sterile cocktail stick was used to scrape mycelium and spores from the surface of not more than 7 day old cultures, which were then mixed in a final volume of $50 \mu \mathrm{l}$ with 250 pmol of each oligonucleotide primer, $200 \mu \mathrm{M}$ dNTPs, $10 \%$ $(\mathrm{v} / \mathrm{v})$ of commercial polymerase buffer, $10 \%$ (v/v) DMSO, $1 \%(\mathrm{v} / \mathrm{v})$ of the non-ionic surfactant Nonidet P40 (BDH/ Merck) and 2.5 units of polymerase (Herculase Enhanced DNA polymerase, Stratagene). Thermal cycling began with a hot start at $95^{\circ} \mathrm{C}$ for 10 minutes before additional amplification of 35 cycles: denaturation at $95^{\circ} \mathrm{C}$ for 1 minute, annealing at $55^{\circ} \mathrm{C}$ for 1 minute (for the universal primers this was at $65^{\circ} \mathrm{C}$ ) followed by 2 minutes extension per kbp of template at $72^{\circ} \mathrm{C}$. A final tailoring step of amplification for 10 minutes was included and products were visualised by staining with ethidium bromide $(0.1$ $\mu \mathrm{g} / \mathrm{ml}$ ) following separation by $1 \%(\mathrm{w} / \mathrm{v})$ agarose gel electrophoresis in TAE buffer.

The in situ colony PCR technique we have described resulted in successful amplification of template DNA (Figure 1). As expected, when oligonucleotide primers specific for the family Actomycetales were used, a $900 \mathrm{bp}$ PCR product could be detected for all of the test strain (Figure 1A). Likewise, when oligonucleotide primers specific for the genus Nonomuria were tested, a $450 \mathrm{bp}$ product could only be detected using Nonomuria spp. templates and not for any of the other actinomycetes (Figure 1B). This PCR technique can be used as a bioprospecting strategy, allowing large numbers of natural isolates to be screened using genus specific primers building up a collection of rare actinomycetes such as Nonomuria spp. to be used for future natural product screening campaigns. This technique also offers the possibility to prescreen natural product collections for specific biosynthetic genes. Here we used oligonucleotide primers to amplify a gene involved in erythromycin biosynthesis, and as expected this gene could only be detected in the erythromycin producer S. erythraea NRRL 2338 (Figure 1C). We believe the success of these 
Fig. 1. Colony PCR amplification of actinomycete DNA using A) universal primers, B) genus specific primers and $\mathrm{C}$ ) primers specific for modular polyketide biosynthesis.

\section{A}
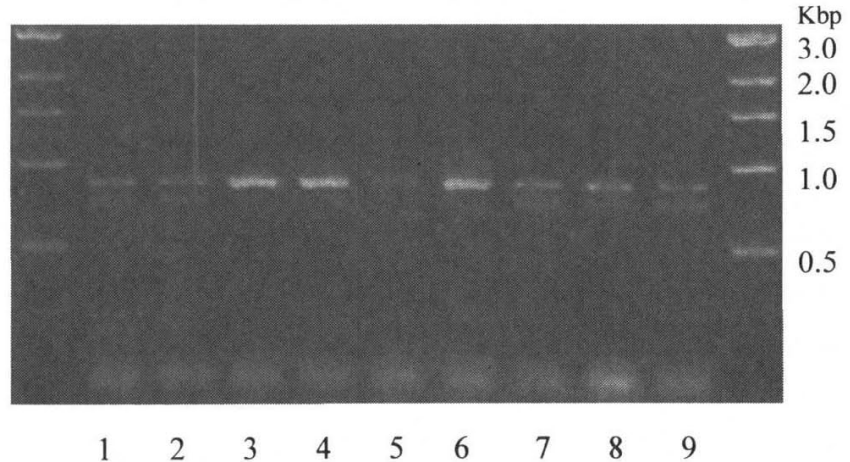

B

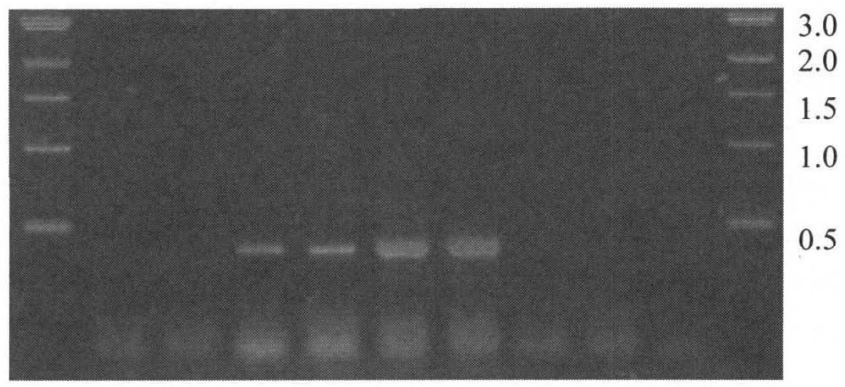

$\begin{array}{lllllllll}1 & 2 & 3 & 4 & 5 & 6 & 7 & 8 & 9\end{array}$

C

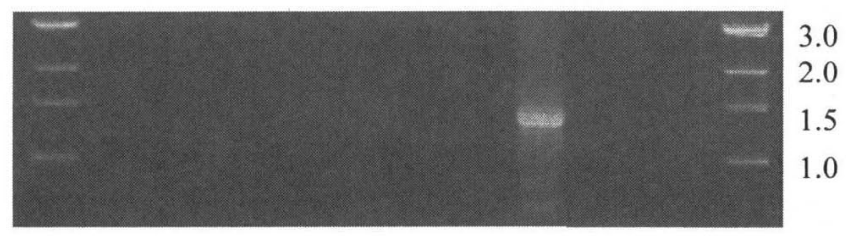

$\begin{array}{lllllllll}1 & 2 & 3 & 4 & 5 & 6 & 7 & 8 & 9\end{array}$

Lane 1 Amycolatopsis mediterranea NCIMB 9613, Lane 2 Mycobacterium avium (clinical isolate), Lane 3 Nonomuraea pusilla NCIMB 11116 , Lane 4 Nonomuraea roseoviolacea subsp. roseoviolacea NCIMB 11117 ,

Lane 5 Nonomuraea ferruginea NCIMB 11630, Lane 6 Nonomuraea roseola NCIMB 11632, Lane 7 Saccharopolyspora erythraea NRRL 2338, Lane 8 Streptomyces antibioticus NCIMB 11506, Lane 9 Streptomyces cinnamonensis NCIMB 12604. techniques is due to heating biomass in the presence of a non-ionic surfactant such as Nonidet P40, allowing sufficient cell wall disruption to occur for the release of DNA template. We did observe that this was dependent on the age of the culture and consistent results could only be obtained on cultures no more than 7 days old.

This report describes colony PCR amplification of high $\mathrm{G}+\mathrm{C}$ content DNA from a range of actinomycete genera. The method we describe seems to have an advantage over previously described protocols ${ }^{3)}$ since it relies upon the use of a non-ionic surfactant to efficiently promote lysis of actinomycete cell walls and enhance DNA polymerase activity. Our approach allows the rapid identification of different genera by the amplification of $16 \mathrm{~S}$ rRNA gene sequences. In addition, we also demonstrated the identification of strains carrying DNA encoding proteins associated with specific metabolic pathways, for example, in polyketide biosynthesis.

\section{Acknowledgments}

This work was funded by grants awarded to PFL from The Wellcome Trust (\# 064197), The Society for General Microbiology (ref: vac2002/18) and The Central Research Fund, University of London (ref: AR/CRF/B).

Esther W. N. Gathogo Alastair C. W. Waugh Nataša PERIĆ

MARIA B. REDPATH

PAUL F. LONG

The School of Pharmacy University of London 29/39 Brunswick Square London, WC1N 1 AX United Kingdom

(Received November 5, 2002)

\section{References}

1) Waugh, A. C. W. \& P. F. Long: Prospects for generating new antibiotics. Sci. Prog. 85(1): 73 88, 2002

2) ZhANG, Z.; Y. WANG \& J. RUAN: Reclassification of Thermomonospora and Microtetraspora. Int. J. Syst. Bacteriol. 48: 411 422, 1998

3) Ishikawa, J.; N. Tsuchizaki, M. Yoshida, D. Ishiyama \& K. HotTA: Colony PCR detection of specific DNA sequences in actinomycetes. Actinomycetologica 14(1): $1 \sim 5,2000$

* Corresponding author: paul.long@ams1.ulsop.ac.uk 